\title{
Hückel-Hubbard-Ohno modeling of $\pi$-bonds in ethene and ethyne with application to trans-polyacetylene
}

\author{
Máte Timár ${ }^{1}$, Gergely Barcza ${ }^{1}$, Florian Gebhard ${ }^{2},{ }^{*}$ Libor $_{\text {Veis }}{ }^{3}{ }^{\dagger}$ and Örs Legeza ${ }^{1 \ddagger}$ \\ ${ }^{1}$ Strongly Correlated Systems Lendület Research Group, Institute for Solid State Physics and Optics, \\ MTA Wigner Research Centre for Physics, P.O. Box 49, H-1525 Budapest, Hungary \\ ${ }^{2}$ Fachbereich Physik, Philipps-Universität Marburg, D-35032 Marburg, Germany and \\ ${ }^{3}$ Dept. of Theoretical Chemistry, J. Heyrovský Institute of Physical Chemistry, \\ Academy of Sciences of the Czech Republic, v.v.i, Dolejškova 3, 18223 Prague 8, Czech Republic
}

(Dated: August 23, 2018)

\begin{abstract}
Quantum chemistry calculations provide the potential energy between two carbon atoms in ethane $\left(\mathrm{H}_{3} \mathrm{C}-\mathrm{CH}_{3}\right)$, ethene $\left(\mathrm{H}_{2} \mathrm{C}=\mathrm{CH}_{2}\right)$, and ethyne $(\mathrm{HC} \equiv \mathrm{CH})$ as a function of the atomic distance. Based on the energy function for the $\sigma$-bond in ethane, $V_{\sigma}(r)$, we use the Hückel model with HubbardOhno interaction for the $\pi$ electrons to describe the energies $V_{\sigma \pi}(r)$ and $V_{\sigma \pi \pi}(r)$ for the $\sigma \pi$ double bond in ethene and the $\sigma \pi \pi$ triple bond in ethyne, respectively. The fit of the force functions shows that the Peierls coupling can be estimated with some precision whereas the Hubbard-Ohno parameters are insignificant at the distances under consideration. We apply the Hückel-HubbardOhno model to describe the bond lengths and the energies of elementary electronic excitations of trans-polyacetylene, $(\mathrm{CH})_{n}$, and adjust the $\sigma$-bond potential for conjugated polymers.
\end{abstract}

PACS numbers: $71.20 . \mathrm{Rv}, 36.20 . \mathrm{Kd}, 31.15 . \mathrm{vn}$

\section{INTRODUCTION}

Electronic structure calculations for small molecules can be performed with very good accuracy using quantum chemistry methods. ${ }^{1,2}$ However, the computational cost rises strongly with the number of valence electrons, and macromolecules with hundreds of delocalized valence electrons cannot be treated using accurate ab-initio quantum chemistry methods. In such a situation, models with adjustable parameters are analyzed to describe the ground state and excited states approximately. ${ }^{3,4}$

An example are $\pi$-conjugated polymers such as polyacetylene (PA) or polydiacetylene (PDA). Typically, the backbone made of $\sigma$-bonds is treated in the adiabatic approximation, and only the $\pi$-electrons are included explicitly in the many-particle model. ${ }^{3,4}$ The electrons' itineracy is described by the Hückel (tight-binding) Hamiltonian, and their Coulomb repulsion is approximated by the Pariser-Parr-Pople (PPP) interaction. Recently, we reproduced the optical properties of PDA using a tightbinding model with a Hubbard-Ohno parameterization of the PPP interaction between the $\pi$ electrons. ${ }^{5}$ However, the applicability of Hückel-PPP models for $\pi$-conjugated systems has been put into question ${ }^{6}$ because the PPP model only includes density-type interactions and ignores bond-charge repulsion terms that could be important for polymers; for a thorough discussion, see Refs. [3,7].

Another point at issue in conjugated polymers is the size of the 'spring constant' $K_{\sigma}^{\mathrm{PA}}$ that parameterizes the strength of the $\sigma$-bond. In Ref. [8], the value $K_{\sigma}^{\mathrm{PA}, 1}=$ $31 \mathrm{eV} / \AA^{2}$ was proposed for PA, close to the value for the carbon-carbon bond in ethane, $D_{1}^{\sigma, \exp }=27.2 \mathrm{eV} / \AA^{2}$ at bond length $r_{\sigma}^{\exp }=1.536 \AA .^{9,10}$ In contrast, in Ref. [11] a substantially larger value, $K_{\sigma}^{\mathrm{PA}, 2}=46 \mathrm{eV} / \AA^{2}$, was put forward, closer to empirical values for benzene, $K_{\sigma}^{\text {ben }}=$ $41.3 \mathrm{eV} / \AA^{2}$ with bond length $r_{0}=1.4 \AA .^{12}$ The larger value is related to the tendency of bonds to become stiffer at smaller atomic distances, ${ }^{13}$ and the average bond length in polyacetylene is the same as that in benzene. $^{14}$

Apparently, Hückel-PPP models can also be applied to small molecules with $\pi$ bonds so that the applicability and accuracy of the model description can be tested against results from quantum chemistry. In this work, we use the two-site Hubbard model to describe the $\pi$ bonds in ethene $\left(\mathrm{H}_{2} \mathrm{C}=\mathrm{CH}_{2}\right)$, and ethyne $(\mathrm{HC} \equiv \mathrm{CH})$. We find that the Hückel approach provides a reasonably accurate description of the $\pi$-bonds. The application to

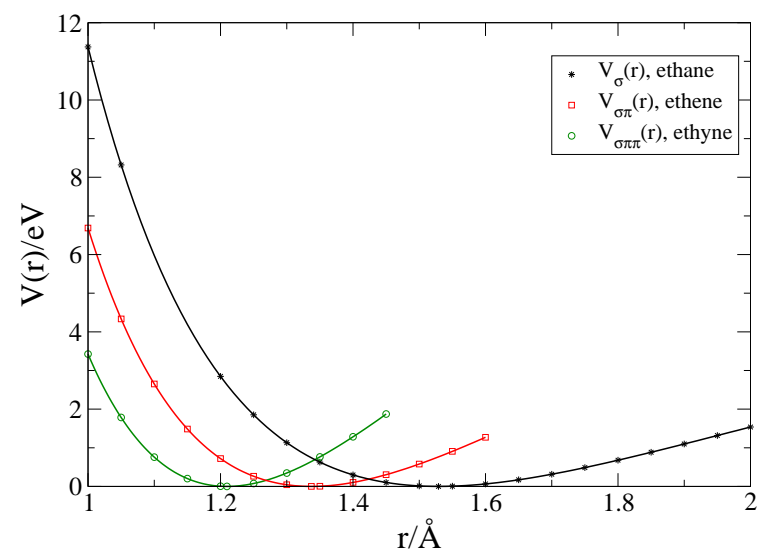

FIG. 1. (Color online) Ground-state energy in electron volts of ethane $\left(\mathrm{H}_{3} \mathrm{C}-\mathrm{CH}_{3}\right), V_{\sigma}(r)$, ethene $\left(\mathrm{H}_{2} \mathrm{C}=\mathrm{CH}_{2}\right.$, ethylene), $V_{\sigma \pi}(r)$, and ethyne ( $\mathrm{HC} \equiv \mathrm{CH}$, acetylene), $V_{\sigma \pi \pi}(r)$, as a function of the carbon-carbon distance $r$ in units of $\AA$. The energies are shifted by their values at the energy minimum. The carbon-hydrogen bonds are fixed at their positions for the equilibrium structure. 
(a)

\begin{tabular}{|c|c|c|c|c|c|}
\hline \multicolumn{2}{|c|}{$\mathrm{H}_{3} \mathrm{C}-\mathrm{CH}_{3}$} & \multicolumn{2}{|c|}{$\mathrm{H}_{2} \mathrm{C}=\mathrm{CH}_{2}$} & \multicolumn{2}{|c|}{$\mathrm{HC} \equiv \mathrm{CH}$} \\
\hline$r / \AA$ & $V_{\sigma}(r) / \mathrm{eV}$ & $r / \AA$ & $V_{\sigma \pi}(r) / \mathrm{eV}$ & $r / \AA$ & $V_{\sigma \pi \pi}(r) / \mathrm{eV}$ \\
\hline 1.0000 & 10.8084 & 1.0000 & 6.56351 & 1.0000 & 3.42482 \\
\hline 1.0500 & 7.89061 & 1.0500 & 4.25500 & 1.0500 & 1.78346 \\
\hline & & 1.1000 & 2.60229 & 1.1000 & 0.75783 \\
\hline & & 1.1500 & 1.45774 & 1.1500 & 0.20269 \\
\hline 1.2000 & 2.68283 & 1.2000 & 0.70626 & $\begin{array}{l}1.2000 \\
1.2097\end{array}$ & $\begin{array}{l}0.00484 \\
0\end{array}$ \\
\hline 1.2500 & 1.74053 & 1.2500 & 0.25796 & 1.2500 & 0.07592 \\
\hline 1.3000 & 1.06070 & $\begin{array}{l}1.3000 \\
1.3372\end{array}$ & $\begin{array}{l}0.04249 \\
0\end{array}$ & 1.3000 & 0.34680 \\
\hline 1.3500 & 0.58770 & 1.3500 & 0.00464 & 1.3500 & 0.76329 \\
\hline 1.4000 & 0.27742 & 1.4000 & 0.10095 & 1.4000 & 1.28284 \\
\hline 1.4500 & 0.09475 & 1.4500 & 0.29714 & 1.4500 & 1.87198 \\
\hline 1.5000 & 0.01163 & 1.5000 & 0.56612 & & \\
\hline 1.5290 & 0 & & & & \\
\hline 1.5500 & 0.00563 & 1.5500 & 0.88651 & & \\
\hline 1.6000 & 0.05877 & 1.6000 & 1.24145 & & \\
\hline 1.6500 & 0.15668 & & & & \\
\hline 1.7000 & 0.28788 & & & & \\
\hline 1.7500 & 0.44322 & & & & \\
\hline 1.8000 & 0.61542 & & & & \\
\hline 1.8500 & 0.79872 & & & & \\
\hline 1.9000 & 0.98856 & & & & \\
\hline 1.9500 & 1.18131 & & & & \\
\hline 2.0000 & 1.37414 & & & & \\
\hline
\end{tabular}

(b)

\begin{tabular}{|c|c|c|c|c|c|}
\hline \multicolumn{2}{|c|}{$\mathrm{H}_{3} \mathrm{C}-\mathrm{CH}_{3}$} & \multicolumn{2}{|c|}{$\mathrm{H}_{2} \mathrm{C}=\mathrm{CH}_{2}$} & \multicolumn{2}{|c|}{$\mathrm{HC} \equiv \mathrm{CH}$} \\
\hline$r / \AA$ & $V_{\sigma}(r) / \mathrm{eV}$ & $r / \AA$ & $V_{\sigma \pi}(r) / \mathrm{eV}$ & $r / \AA$ & $V_{\sigma \pi \pi}(r) / \mathrm{eV}$ \\
\hline 1.0000 & 11.3730 & 1.0000 & 6.68729 & 1.0000 & 3.42561 \\
\hline 1.0500 & 8.31871 & 1.0500 & 4.33523 & 1.0500 & 1.78388 \\
\hline & & 1.1000 & 2.65165 & 1.1000 & 0.75802 \\
\hline & & 1.1500 & 1.48570 & 1.1500 & 0.20275 \\
\hline 1.2000 & 2.84798 & 1.2000 & 0.72002 & $\begin{array}{l}1.2000 \\
1.2097\end{array}$ & $\begin{array}{l}0.00485 \\
0\end{array}$ \\
\hline 1.2500 & 1.85257 & 1.2500 & 0.26309 & 1.2500 & 0.07594 \\
\hline 1.3000 & 1.13216 & $\begin{array}{l}1.3000 \\
1.3372\end{array}$ & $\begin{array}{l}0.04336 \\
0\end{array}$ & 1.3000 & 0.34691 \\
\hline 1.3500 & 0.62916 & 1.3500 & 0.00474 & 1.3500 & 0.76358 \\
\hline 1.4000 & 0.29792 & 1.4000 & 0.10310 & 1.4000 & 1.28340 \\
\hline 1.4500 & 0.10209 & 1.4500 & 0.30366 & 1.4500 & 1.87290 \\
\hline 1.5000 & 0.01258 & 1.5000 & 0.57893 & & \\
\hline 1.5290 & 0 & & & & \\
\hline 1.5500 & 0.00609 & 1.5500 & 0.90718 & & \\
\hline 1.6000 & 0.06391 & 1.6000 & 1.27127 & & \\
\hline 1.6500 & 0.17101 & & & & \\
\hline 1.7000 & 0.31534 & & & & \\
\hline 1.7500 & 0.48722 & & & & \\
\hline 1.8000 & 0.67891 & & & & \\
\hline 1.8500 & 0.88423 & & & & \\
\hline 1.9000 & 1.09820 & & & & \\
\hline 1.9500 & 1.31686 & & & & \\
\hline 2.0000 & 1.53703 & & & & \\
\hline
\end{tabular}

TABLE I. Ground-state energy of ethane $\left(\mathrm{H}_{3} \mathrm{C}-\mathrm{CH}_{3}\right), V_{\sigma}(r)$, ethene (ethylene, $\left.\mathrm{H}_{2} \mathrm{C}=\mathrm{CH}_{2}\right), V_{\sigma \pi}(r)$, and ethyne (acetylene, $\mathrm{HC} \equiv \mathrm{CH}), V_{\sigma \pi \pi}(r)$, in units of $\mathrm{eV}$ as a function of the carbon-carbon distance $r$ in units of $\AA$. The energies are shifted by a constant so that the potential is zero at the equilibrium distance. (a) The whole molecule is relaxed; (b) The carbon-hydrogen bonds are fixed at their positions for the equilibrium structure.

trans-PA shows, however, that some adjustments in the $\sigma$-bond spring constant are necessary to reproduce the experimentally observed bond lengths.

Our work is organized as follows. In Sect. II we use data from quantum chemistry calculations to express the ground-state energies $V_{\sigma}(r), V_{\sigma \pi}(r)$, and $V_{\sigma \pi \pi}(r)$ for $\sigma$-bonds and $\pi$-bonds in ethane, ethene, and ethyne. In Sect. III we use the Hückel-Hubbard-Ohno Hamiltonian to model the energy of the $\pi$-bonds in ethene and ethyne. We shall see that this is possible with an accuracy of about 5\%. However, the Hubbard-Ohno parameters for the Coulomb interaction remain essentially undetermined. In Sect. IV, to test and adjust our parameter set, we determine the bond lengths and the energies of elementary electronic excitations in trans-PA. Short conclusions, Sect. V, close our presentation.

\section{ENERGY OF CARBON SINGLE, DOUBLE, AND TRIPLE BONDS}

\section{A. Data from quantum chemistry}

We employ the quantum chemistry code MoLPRO ${ }^{15}$ using the $\operatorname{CCSD}(\mathrm{T})$ method with the cc-pVTZ basis. ${ }^{16} \mathrm{We}$ calculate the energy of the molecules ethane $\left(\mathrm{H}_{3} \mathrm{C}-\mathrm{CH}_{3}\right)$, ethene (a.k.a. ethylene, $\mathrm{H}_{2} \mathrm{C}=\mathrm{CH}_{2}$ ), and ethyne (a.k.a. acetylene, $\mathrm{HC} \equiv \mathrm{CH}$ ) as a function of the carbon-carbon distance $r$. The data for the energies are listed in Table I. Similar calculations have been done recently for ethyne, ${ }^{17}$ and for $\mathrm{C}_{2} \mathrm{H}_{2 n}$ for $n=0,1,2,3 .{ }^{18,19}$ We can (a) choose to relax the whole molecule, or (b) to fix the carbon-hydrogen bonds at their positions for the equilibrium structure. The data from quantum chemistry in Table I show that the changes are marginal for ethyne and still very small for ethene. This could be expected because the carbon-hydrogen bonds in the corresponding linear/planar structures do not interfere when we stretch or shrink the carbon-carbon bond. For ethane with its three-dimensional structure, however, the discrepancies in energy are noticeable. Since we are interested in the properties of the $\sigma$-bond, we later use the data for fixed carbon-hydrogen bonds and plot the corresponding energies from Table I(b) as a function of distance in Fig. 1.

\section{B. Polynomial parameterization}

First, we compare the bond lengths with the experimental data. We find for ethane (one $\sigma$-bond), ethene (one $\sigma$-bond plus one $\pi$-bond), and ethyne (one $\sigma$-bond 
(a)

\begin{tabular}{|c|c|c|c|}
\hline \multirow{2}{*}{\multicolumn{2}{|c|}{$\mathrm{H}_{3} \mathrm{C}-\mathrm{CH}_{3}$}} & \multirow{2}{*}{$\begin{array}{c}\mathrm{H}_{2} \mathrm{C}=\mathrm{CH}_{2} \\
D_{l}^{\sigma \pi}\left[\mathrm{eV} / \AA^{l+1}\right]\end{array}$} & $\mathrm{HC} \equiv \mathrm{CH}$ \\
\hline & {$\left[\mathrm{eV} / \AA^{l+1}\right]$} & & $D_{l}^{\sigma \pi \pi} \quad\left[\mathrm{eV} / \AA^{l+1}\right.$ \\
\hline$D_{1}^{\sigma}$ & 26.3496 & 57.4115 & $D_{1}^{\sigma \pi \pi}$ \\
\hline$D_{2}^{\sigma}$ & -69.9253 & $D_{2}^{\sigma \pi}-162.4340$ & -294.7982 \\
\hline$D_{3}^{\sigma}$ & 104.1792 & $D_{3}^{\sigma \pi} \quad 259.2015$ & 482.5564 \\
\hline$D_{4}^{\sigma}$ & -119.1238 & $D_{4}^{\sigma \pi}-336.1312$ & $D_{4}^{\sigma \pi \pi}-656.0314$ \\
\hline$D_{5}^{\sigma}$ & 105.2503 & 284.6613 & 664.8695 \\
\hline$D_{6}^{\sigma}$ & -70.7506 & $D_{6}^{\sigma \pi}-116.8712$ & \\
\hline & 135.5519 & $D_{7}^{\sigma \pi} \quad 816.7895$ & \\
\hline$D_{8}^{\sigma}$ & -146.0841 & & \\
\hline$D_{9}^{\sigma}$ & -86.5683 & & \\
\hline
\end{tabular}

(b)

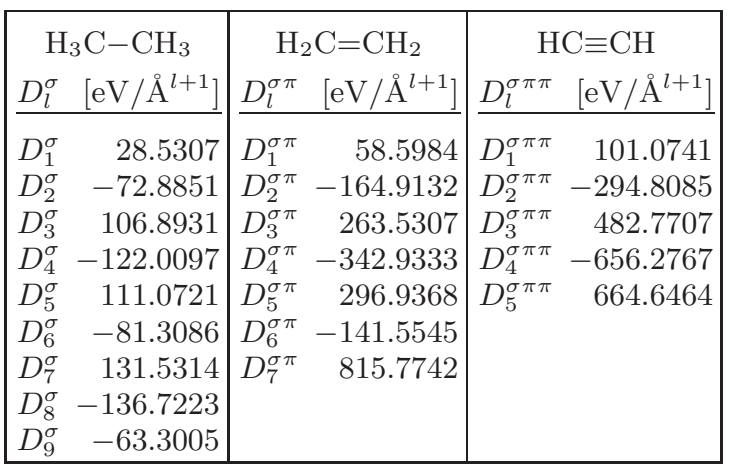

TABLE II. Parameters $D_{l}(l \leq 9)$ for the force fields $F_{\sigma}(r), F_{\sigma \pi}(r)$, and $F_{\sigma \pi \pi}(r)$, of ethane, ethene, and ethyne, respectively. (a) The whole molecule is relaxed; (b) The carbon-hydrogen bonds are fixed at their positions for the equilibrium structure.

plus two $\pi$-bonds),

$$
\begin{aligned}
r_{\sigma}=1.5290 \AA, \quad r_{\sigma}^{\exp }=1.5360 \AA & {[9], } \\
r_{\sigma \pi}=1.3372 \AA, \quad r_{\sigma \pi}^{\exp }=1.3390 \AA & {[9], } \\
r_{\sigma \pi \pi}=1.2097 \AA, \quad r_{\sigma \pi \pi}^{\exp }=1.203 \AA & {[20], }
\end{aligned}
$$

in very good agreement with the experimental values, with deviations of less than $0.5 \%$.

Next, we express the energies $V_{\mathrm{e}}(r)$ as a Taylor series around the equilibrium distances $r_{\mathrm{e}}$,

$$
V_{\mathrm{e}}(r)=V_{0}^{\mathrm{e}}+\sum_{n=2}^{10} \frac{D_{n-1}^{\mathrm{e}}}{n}\left(r-r_{\mathrm{e}}\right)^{n},
$$

where $\mathrm{e}=\sigma, \sigma \pi, \sigma \pi \pi$ for the three molecules, and $V_{0}^{\mathrm{e}}$ is independent of $r$. In the following, we focus on the force fields $F_{\mathrm{e}}(r)=-V_{\mathrm{e}}^{\prime}(r)$,

$$
F_{\mathrm{e}}(r)=-\sum_{n=1}^{9} D_{n}^{\mathrm{e}}\left(r-r_{\mathrm{e}}\right)^{n}
$$

because they enter the optimization equations. The parameter sets for ethane, ethene, and ethyne are collected in Table II.

One of the vibrational modes in ethane, ethene, and ethyne with reciprocal wave length (wave number) $1 / \lambda^{\mathrm{e}}$ can be assigned to a bare carbon-carbon stretch mode. They can be compared to the values from quantumchemistry calculations,

$$
\begin{aligned}
1 / \lambda_{\sigma}=1013 \mathrm{~cm}^{-1}, \quad 1 / \lambda_{\sigma}^{\exp }=992.9 \mathrm{~cm}^{-1} & {[10], } \\
1 / \lambda_{\sigma \pi}=1671 \mathrm{~cm}^{-1}, \quad 1 / \lambda_{\sigma \pi}^{\exp }=1623 \mathrm{~cm}^{-1} & {[21], } \\
1 / \lambda_{\sigma \pi \pi}=2000 \mathrm{~cm}^{-1}, 1 / \lambda_{\sigma \pi \pi}^{\exp }=1974 \mathrm{~cm}^{-1} & {[22] . }
\end{aligned}
$$

The experimental values in eq. (4) are in very good agreement with the results from quantum chemistry, with deviations of less than $3 \%$. This is the desired accuracy of the fit to a Hückel-Hubbard-Ohno model.

The normal-mode frequencies given in eq. (4) correspond to vibrational modes that involve the movement of all atoms in the molecule. Instead, one may want to invoke a simpler oscillator model with fixed carbonhydrogen bonds, a single force constant $\tilde{D}_{1}^{\mathrm{e}}$, and an effective mass $m^{\mathrm{e}}=(12+n) u / 2$ (atomic mass unit: $u=1.66054 \cdot 10^{-27} \mathrm{~kg} ; n=1,2,3$ for ethane, ethene, ethyne). ${ }^{11}$ Then, the force constant obeys

$$
\tilde{D}_{1}^{\mathrm{e}}=m^{\mathrm{e}} c^{2}\left(\frac{2 \pi}{\lambda_{\mathrm{CC}}^{\mathrm{e}}}\right)^{2} .
$$

A fit to the experimental data for ethane, ethene, and ethyne then leads to

$$
\begin{aligned}
\tilde{D}_{1}^{\sigma} & =27.2 \mathrm{eV} / \AA^{2}, \quad D_{1}^{\sigma}=26.3 \mathrm{eV} / \AA^{2}, \\
\tilde{D}_{1}^{\sigma \pi} & =67.8 \mathrm{eV} / \AA^{2}, \quad D_{1}^{\sigma \pi}=57.4 \mathrm{eV} / \AA^{2}, \\
\tilde{D}_{1}^{\sigma \pi \pi} & =93.1 \mathrm{eV} / \AA^{2}, D_{1}^{\sigma \pi \pi}=101.0 \mathrm{eV} / \AA^{2},
\end{aligned}
$$

where we used $1 \mathrm{~N} / \mathrm{m}=0.01 \mathrm{mdyn} / \AA=1 / 16.022 \mathrm{eV} / \AA^{2}$ for the conversion of units. The comparison shows that oscillator models with a single 'spring constant' shall employ values $\tilde{D}_{1}$ that deviate from the bare values $D_{1}$ from quantum chemistry calculations by $10 \%-15 \%$.

\section{HÜCKEL-HUBBARD-OHNO MODEL}

\section{A. Hamiltonian for a $\pi$-bond}

We model a $\pi$-bond by a two-site single-orbital HückelHubbard-Ohno model,

$$
\begin{aligned}
\hat{H}_{\pi}(r)= & -t(r)\left(\hat{c}_{1, \uparrow}^{\dagger} \hat{c}_{2, \uparrow}+\hat{c}_{2, \uparrow}^{\dagger} \hat{c}_{1, \uparrow}+\hat{c}_{1, \downarrow}^{\dagger} \hat{c}_{2, \downarrow}+\hat{c}_{2, \downarrow}^{\dagger} \hat{c}_{1, \downarrow}\right) \\
& +U\left(\hat{n}_{1, \uparrow} \hat{n}_{1, \downarrow}+\hat{n}_{2, \uparrow} \hat{n}_{2, \downarrow}\right) \\
& +V(r)\left(\hat{n}_{1}-1\right)\left(\hat{n}_{2}-1\right)
\end{aligned}
$$

where $\hat{c}_{l, \sigma}^{\dagger}\left(\hat{c}_{l, \sigma}\right)$ creates (annihilates) an electron with spin $\sigma=\{\uparrow, \downarrow\}$ on carbon atom $l=1,2$. Moreover, $\hat{n}_{l, \sigma}=\hat{c}_{l, \sigma}^{\dagger} \hat{c}_{l, \sigma}$ counts the number of $\sigma$-electrons on carbon atom $l$ and $\hat{n}_{l}=\hat{n}_{l, \uparrow}+\hat{n}_{l, \downarrow}$. We assume that the $\sigma$-bond is rigid and contributes the potential $V_{\sigma}(r)$ to the energy.

The Hamiltonian parametrically depends on the distance $r$ between the carbon atoms. The electron transfer 
parameter decreases exponentially as a function of distance,

$$
t_{\alpha}(r)=t_{0} \exp \left(-\alpha\left(r-r_{0}\right) / t_{0}\right),
$$

with $t_{\alpha}\left(r_{0}\right)=t_{0} \equiv 2.5 \mathrm{eV}$ at distance $r=r_{0} \equiv 1.4 \AA$. The strength of the Peierls coupling $\alpha$ and the values for the Coulomb interaction $U$ and $V$ are fit parameters. The distance-dependence of the density-density interaction is given by the Ohno expression ${ }^{3,4}$

$$
V(r)=\frac{V}{\sqrt{1+\beta(r / \AA)^{2}}}, \beta=\left(\frac{V}{14.397 \mathrm{eV}}\right)^{2} .
$$

The Ohno form guarantees that, at large distances, the electrons interact via their unscreened Coulomb interaction, $e^{2}=14.397 \mathrm{eV \AA}$.

The ground-state energy of the Hückel-Hubbard-Ohno model is denoted by $E_{\pi}(r, \alpha, U, V)$. The two-site model is readily diagonalized, ${ }^{5}$

$E_{\pi}(r, \alpha, U, V)=\frac{U-V(r)}{2}-\sqrt{\frac{(U-V(r))^{2}}{4}+4\left[t_{\alpha}(r)\right]^{2}}$

Up to a constant energy shift, $V_{\sigma}(r)+E_{\pi}(r, \alpha, U, V)$ defines the Hückel-Hubbard-Ohno approximation to the energy function for ethene, $V_{\sigma \pi}(r)$.

For ethyne, we further assume that the two $\pi$-bonds are independent of each other. An explicit calculation of the ground-state energy of a two-site model with four orbitals per site shows that, for moderate Coulomb interactions, the ground-state energy can indeed be approximated as the sum of two independent two-orbital models.

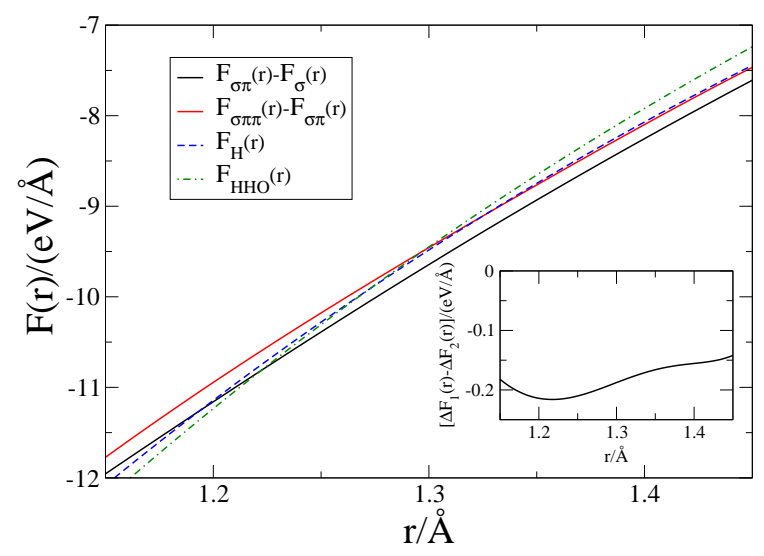

FIG. 2. (Color online) Force function differences $\Delta F_{1}(r)=$ $F_{\sigma \pi}(r)-F_{\sigma}(r), \Delta F_{2}(r)=F_{\sigma \pi \pi}(r)-F_{\sigma \pi}(r)$ due to the $\pi$ bonds in ethene and in ethyne for carbon distances $1.15 \AA<$ $r<1.45 \AA$. The force function differences are not the same, they deviate from each other by about $0.2 \mathrm{eV}$ for $1.15 \AA<$ $r<1.45 \AA$, as seen in the inset. The dashed lines show the fit from the Hückel model, $F_{\mathrm{H}}(r)=-E_{\pi}^{\prime}(r, 4.035 \mathrm{eV} / \AA, 0,0)$ and from the Hückel-Hubbard-Ohno model, $F_{\mathrm{HHO}}(r)=$ $-E_{\pi}^{\prime}(r, 4.119 \mathrm{eV} / \AA, 6 \mathrm{eV}, 3 \mathrm{eV})$.
Therefore, the Hückel-Hubbard-Ohno approach predicts

$$
V_{\sigma \pi}(r)-V_{\sigma}(r)+C_{1}=V_{\sigma \pi \pi}(r)-V_{\sigma \pi}(r)+C_{2} .
$$

The quality of this hypothesis can be tested by comparing the corresponding differences in the force functions, $\Delta F_{1}(r)=F_{\sigma \pi}(r)-F_{\sigma}(r)$ and $\Delta F_{2}(r)=F_{\sigma \pi \pi}(r)-$ $F_{\sigma \pi}(r)$.

In Fig. 2 we show the two force function differences $\Delta F_{i}(r)$ for carbon-carbon distances $1.15 \AA<r<1.45 \AA$. As is seen from the inset, they differ by about $0.2 \mathrm{eV} / \AA$. Similarly, the Hückel force field $F_{\mathrm{H}}(r)$ at $\alpha=4.035 \mathrm{eV}$ and the Hückel-Hubbard-Ohno force field $F_{\mathrm{HHO}}(r)$ at $\alpha=4.119 \mathrm{eV}$ and $U=2 \mathrm{~V}=6 \mathrm{eV}$ deviate from $\Delta F_{i}(r)$ by several tenth of an $\mathrm{eV} / \AA$. Since $\Delta F_{i}(r)$ is of the order of $10 \mathrm{eV} / \AA$, the Hückel-Hubbard-Ohno description of the $\pi$-bonds in ethene and ethyne is accurate within a few percent which is of the same order of magnitude as the accuracy of the quantum chemistry data for the vibrational frequencies. Therefore, the Hückel-HubbardOhno description is accurate enough to match the quality of the quantum chemistry data. For a further improvement, one may refine the expression (8) for the distancedependence of the electron transfer parameter. ${ }^{23}$

\section{B. Parameter optimization}

Since the force functions $\Delta F_{i}(r)$ differ, we cannot determine $E_{\pi}(r, \alpha, U, V)$ from eq. (11) as the difference between $V_{\sigma}(r)$ and $V_{\sigma \pi}(r)$. Instead, we have to set up an optimization scheme. We define the cost function (all lengths in $\AA$, all energies in $\mathrm{eV}$ )

$$
\begin{aligned}
W(\alpha, U, V)= & \int_{1.15}^{1.45} \mathrm{~d} r\left(F_{\sigma \pi}(r)-F_{\sigma}(r)+E_{\pi}^{\prime}(r)\right)^{2} \\
& +\int_{1.15}^{1.45} \mathrm{~d} r\left(F_{\sigma \pi \pi}(r)-F_{\sigma \pi}(r)+E_{\pi}^{\prime}(r)\right)^{2}
\end{aligned}
$$

where $E_{\pi}^{\prime}(r)=\left(\mathrm{d} E_{\pi}(r, \alpha, U, V)\right) /(\mathrm{d} r)$ is the negative force field due to the $\pi$-bond in the Hückel-HubbardOhno description. Using this cost function for the parameter optimization, the Hückel-Hubbard-Ohno model will provide a suitable description of the $\pi$-bonds in ethene and ethyne.

As a first step, we seek the optimal value for the bare Hückel model, i.e., we optimize $W(\alpha, 0,0)$. The result is $\alpha^{\mathrm{H}}=4.035 \mathrm{eV} / \AA$. The Hückel model with $\alpha^{\mathrm{H}}$ provides a good description of the $\pi$-bonds, see Fig. 2 . The bare Hückel force field, $F_{\mathrm{H}}(r)=-E_{\pi}^{\prime}(r, 4.035 \mathrm{eV} / \AA, 0,0)$ deviates from the force fields $\Delta F_{1,2}(r)$ by only a few tenth of an $\mathrm{eV} / \AA$. As a consequence, the Hückel model reproduces the bond lengths $r_{\sigma \pi}$ and $r_{\sigma \pi \pi}$ and the parameters $D_{1}^{\sigma \pi}$ and $D_{1}^{\sigma \pi \pi}$ from quantum chemistry calculations with good accuracy, see Table III.

The success of the bare Hückel model indicates that the Hubbard-Ohno interaction cannot improve the results very much. More importantly, for moderate values 


\begin{tabular}{|c|c|c|c|c|}
\hline & $\frac{r_{\sigma \pi}}{\AA}$ & $\frac{D_{1}^{\sigma \pi}}{\mathrm{eV} / \AA^{2}}$ & $\frac{r_{\sigma \pi \pi}}{\AA}$ & $\frac{D_{1}^{\sigma \pi \pi}}{\mathrm{eV} / \AA^{2}}$ \\
\hline Expe & 1.3390 & & 1.203 & \\
\hline antum Chemistry & 1.3372 & 58.60 & 1.2097 & 01.07 \\
\hline Hückel model $(U=V=0)$ & 1.3403 & 57.28 & 1.2079 & 100.10 \\
\hline Hückel-Hubbard-Ohno model & 1.3417 & 55.66 & 1.2071 & 99.28 \\
\hline
\end{tabular}

TABLE III. Equilibrium distances and force parameters for ethene and ethyne from experiment, quantum chemistry CCSD(T)/cc-pVTZ, the Hückel model with $\alpha=4.035 \mathrm{eV} / \AA$, and the Hückel-Hubbard-Ohno model with $\alpha=4.119 \mathrm{eV} / \AA$, $U=6 \mathrm{eV}, V=3 \mathrm{eV}$.

for $V(V<6 \mathrm{eV})$ and for $r$ of the order of one Angstrøm, the dependence of $V(r)$ on $r$ is fairly small, and the range of acceptable values for $U$ and $V$ is quite large for the optimization functions chosen. Correspondingly, shortrange parameterizations of the PPP potential can give parameter values very different from ours. ${ }^{24}$ Given the finite accuracy of the quantum chemistry data, it is therefore difficult to derive reliable values for $U$ and $V$ from our fits.

We note, however, that $U$ and $V$ strongly influence the size of the single-particle gap and the exciton binding energy in polymers. Therefore, we argue that the Coulomb parameters for short molecules should not differ much from the parameter set $(U=6 \mathrm{eV}, V=3 \mathrm{eV})$ as obtained from our analysis of the PDA spectra. ${ }^{5}$ For this reason, we restrict ourselves to the optimization of $W(\alpha, 6 \mathrm{eV}, 3 \mathrm{eV})$, and obtain $\alpha^{\mathrm{HHO}}=4.119 \mathrm{eV} / \AA$ as the optimal value. As for the bare Hückel model, the HückelHubbard-Ohno model reproduces the bond lengths and force parameters from quantum chemistry calculations with good accuracy, see Table III. The overall agreement with the force functions is also quite good for all carbon-carbon distances $1.15 \AA<r<1.45 \AA$, see Fig. 2 .

\section{APPLICATION TO POLYACETYLENE}

\section{A. Parameters for trans-polyacetylene}

The analysis of the bond energies in our di-carbon molecules permits two conclusions. First, the Peierls coupling should be $\alpha \approx 4 \mathrm{eV} / \AA$ which is somewhat smaller than the value proposed by $\mathrm{Su}$, Schrieffer and Heeger, $\alpha^{\mathrm{SSH}}=4.7 \mathrm{eV} / \AA \AA^{3,25}$ Similar values, with a deviation of $\pm 20 \%$, were generally used for the description of $\pi$ conjugated polymers. ${ }^{4,8,11}$

Second, we can expand the $\sigma$-bond potential around the average bond distance in trans-PA to determine the spring constant $K_{\sigma}^{\mathrm{PA}}$. We Taylor expand the $\sigma$-bond force field $F_{\sigma}(r)$ around $r=r_{0}$,

$$
F_{\sigma}(r)=-\sum_{n=1}^{9} D_{n}^{\sigma}\left(r-r_{\sigma}\right)^{n}=-\sum_{n=0}^{9} K_{\sigma, n}\left(r-r_{0}\right)^{n},
$$

and find $\left(K_{\sigma, n}\right.$ is in units of $\left.\mathrm{eV} / \AA^{n+1}\right)$

$$
\begin{aligned}
& K_{\sigma, 0}=-5.16199, K_{\sigma, 1}=53.8998, K_{\sigma, 2}=-129.282, \\
& K_{\sigma, 3}=193.365, K_{\sigma, 4}=-226.216, K_{\sigma, 5}=234.224, \\
& K_{\sigma, 6}=-252.402, K_{\sigma, 7}=234.716, K_{\sigma, 8}=-63.2203,
\end{aligned}
$$

and $K_{\sigma, 9}=-63.3005 \mathrm{eV} / \AA^{10}=D_{9}^{\sigma}$, see Table II(b). The first term, $-K_{\sigma, 0}=5.2 \mathrm{eV} / \AA>0$, describes the repulsive force of the $\sigma$-bond. It opposes the shrinking of the $\sigma$-bonds in trans-PA from $r_{\sigma}=1.529 \AA$ down to $r_{0}=1.4 \AA$. This repulsive force must be compensated by the binding due to the itinerant $\pi$-electrons.

The second term describes the enhanced spring constant due to the compressed $\sigma$-bond, $K_{\sigma, 1}=54 \mathrm{eV} / \AA^{2}$, so that we should set $K_{\sigma}^{\mathrm{PA}}=K_{\sigma, 1}$. This value can be compared with the literature values used for transpolyacetylene. ${ }^{8,11}$ Our analysis of the $\sigma$-bond potential in ethane, $V_{\sigma}(r)$, supports the larger value derived in Ref. [11], $K_{\sigma}^{\mathrm{PA}, 2}=46 \mathrm{eV} / \AA^{2}$. Moreover, we find $\alpha / K_{\sigma}^{\mathrm{PA}} \approx 0.08 \AA$ which implies that the electron-phonon coupling constant,

$$
\lambda=\frac{2 \alpha^{2}}{\pi t_{0} K_{\sigma}^{\mathrm{PA}}},
$$

is small, $\lambda \approx 0.08 .^{3}$ Therefore, polaronic effects are predicted to be of minor importance for trans-polyacetylene, in contrast to the $\mathrm{SSH}$ picture, $\lambda^{\mathrm{SHH}}=0.2{ }^{25}$

\section{B. Hückel model}

The values for the electron-phonon coupling and the $\sigma$-bond potential $V_{\sigma}(r)$ are important for the theoretical description of the bond lengths in PA. We find that the simple Hückel model cannot account for the dimerization in trans-polyacetylene.

\section{Hamiltonian}

We consider $L$ unit cells with two carbon atoms each. The spin- $1 / 2$ electrons move on sites $l=1,2, \ldots, 2 L$, and periodic boundary conditions apply. The system is half filled, i.e., the number of electrons equals the number of sites, $N_{\uparrow}+N_{\downarrow}=2 L$; the system is paramagnetic, $N_{\uparrow}=N_{\downarrow}=L$. In the Hückel description, the electrons move between neighboring sites,

$$
\begin{aligned}
\hat{T}=-\sum_{\sigma} \sum_{l=1}^{L}[ & t_{\text {odd }}\left(\hat{c}_{2 l-1, \sigma}^{\dagger} \hat{c}_{2 l, \sigma}+\hat{c}_{2 l, \sigma}^{\dagger} \hat{c}_{2 l-1, \sigma}\right) \\
& \left.+t_{\text {even }}\left(\hat{c}_{2 l, \sigma}^{\dagger} \hat{c}_{2 l+1, \sigma}+\hat{c}_{2 l+1, \sigma}^{\dagger} \hat{c}_{2 l, \sigma}\right)\right],
\end{aligned}
$$

where

$$
t_{\text {odd }}=t_{\alpha}\left(r_{\mathrm{s}}\right) \quad, \quad t_{\text {even }}=t_{\alpha}\left(r_{\mathrm{d}}\right)
$$


are the electron transfer matrix elements for the singlebonds and double-bonds, respectively. In this Ansatz we take into account the bond dimerization (Peierls effect). The lengths of the single and double bonds are given by

$$
r_{\mathrm{s}}=r_{\sigma}-s+\Delta \quad, \quad r_{\mathrm{d}}=r_{\sigma}-s-\Delta .
$$

Here, $s$ describes the average bond-length reduction from $r_{\sigma}$ to $r_{\sigma}-s$, and $\Delta$ describes the bond-length alternation.

The total Hamiltonian is given by the sum of the electrons' kinetic energy and the potential energy contribution from $V_{\sigma}(r)$,

$$
\begin{aligned}
\hat{H}^{\mathrm{H}}(s, \Delta) & =\hat{T}(s, \Delta)+L V_{\text {bond }}(s, \Delta) \\
V_{\text {bond }}(s, \Delta) & =V_{\sigma}\left(r_{\sigma}-s+\Delta\right)+V_{\sigma}\left(r_{\sigma}-s-\Delta\right) .
\end{aligned}
$$

At the optimal values of $s$ and $\Delta$, the ground-state energy of $\hat{H}^{\mathrm{H}}$ has its minimum. Experimentally, ${ }^{14}$ we have $r_{\mathrm{s}}=$ $1.44 \AA$ and $r_{\mathrm{d}}=1.36 \AA$ with $\left(r_{\mathrm{s}}+r_{\mathrm{d}}\right) / 2=r_{0}=1.40 \AA$, so that $\Delta_{0}^{\exp }=\left(r_{\mathrm{s}}-r_{\mathrm{d}}\right) / 2=0.04 \AA$ and $s_{0}^{\exp }=r_{\sigma}-r_{0}=$ $0.136 \AA$.

\section{Ground-state energy}

To determine the ground-state energy as a function of $s$ and $\Delta$, we introduce the dimensionless units

$$
\sigma=\frac{s \alpha}{t_{0}} \quad, \quad \delta=\frac{\Delta \alpha}{t_{0}} .
$$

The bare dispersion relation $\epsilon(k)$ and the hybridization function $\Delta(k)$ are given by

$$
\begin{aligned}
\epsilon(k) & =-\left(t_{\text {odd }}+t_{\text {even }}\right) \cos (k)=-2 \tilde{t}_{0} \cos (k) e^{\sigma} \cosh (\delta), \\
\Delta(k) & =\left(t_{\text {even }}-t_{\text {odd }}\right) \sin (k)=2 \tilde{t}_{0} \sin (k) e^{\sigma} \sinh (\delta) .
\end{aligned}
$$

Here, we use the abbreviation $\tilde{t}_{0}=t_{0} \exp \left[-\left(r_{\sigma}-r_{0}\right) \alpha / t_{0}\right]$. The Hückel-Peierls Hamiltonian is diagonal in reciprocal space, ${ }^{25,26}$

$$
\begin{aligned}
\hat{T} & =\sum_{|k| \leq \pi / 2, \sigma} E(k)\left(\hat{a}_{k, \sigma,+}^{\dagger} \hat{a}_{k, \sigma,+}-\hat{a}_{k, \sigma,-}^{\dagger} \hat{a}_{k, \sigma,-}\right), \\
E(k) & =\sqrt{[\epsilon(k)]^{2}+[\Delta(k)]^{2}} \\
& =2 \tilde{t}_{0} e^{\sigma} \sqrt{[\cosh (\delta)]^{2}-[\sin (k)]^{2}}
\end{aligned}
$$

Here, $\pm E(k)$ is the dispersion relation for the upper $(+)$ and lower (-) Peierls band. The ground state is the Peierls insulator,

$$
\left|\Psi_{0}\right\rangle=\prod_{\sigma} \prod_{|k| \leq \pi / 2} \hat{a}_{k, \sigma,-}^{\dagger}|\mathrm{vac}\rangle
$$

where the lower Peierls band is completely filled. Note that the wave numbers $k$ are quantized in units of $2 \pi /(2 L)$ because the chain has $2 L$ sites.

The sum of the electrons' kinetic energy and of the lattice potential energy per unit cell is given by

$$
\begin{aligned}
e_{\text {tot }}(\sigma, \delta) & =T(\sigma, \delta) / L+V_{\text {bond }}\left(t_{0} \sigma / \alpha, t_{0} \delta / \alpha\right) \\
T(\sigma, \delta) / L & =-4 \tilde{t}_{0} e^{\sigma} \int_{-\pi / 2}^{\pi / 2} \frac{\mathrm{d} k}{\pi} \sqrt{[\cosh (\delta)]^{2}-[\sin (k)]^{2}} .
\end{aligned}
$$

A factor of two in the kinetic energy accounts for the spin degeneracy. The minimization with respect to $\sigma$ gives

$$
\begin{aligned}
-\frac{T\left(\sigma_{0}, \delta_{0}\right)}{L}= & 4 \tilde{t}_{0} e^{\sigma_{0}} \int_{-\pi / 2}^{\pi / 2} \frac{\mathrm{d} k}{\pi} \sqrt{\left[\cosh \left(\delta_{0}\right)\right]^{2}-[\sin (k)]^{2}} \\
= & \frac{t_{0}}{\alpha}\left[F_{\sigma}\left(r_{\sigma}-\left(t_{0} / \alpha\right) \sigma_{0}+\left(t_{0} / \alpha\right) \delta_{0}\right)\right. \\
& \left.+F_{\sigma}\left(r_{\sigma}-\left(t_{0} / \alpha\right) \sigma_{0}-\left(t_{0} / \alpha\right) \delta_{0}\right)\right] .
\end{aligned}
$$

The minimization with respect to $\delta$ leads to

$$
\begin{aligned}
\frac{T^{\prime}\left(\sigma_{0}, \delta_{0}\right)}{L}=-4 \tilde{t}_{0} e^{\sigma_{0}} \int_{-\pi / 2}^{\pi / 2} \frac{\mathrm{d} k}{\pi} \frac{\cosh \left(\delta_{0}\right) \sinh \left(\delta_{0}\right)}{\sqrt{\left[\cosh \left(\delta_{0}\right)\right]^{2}-[\sin (k)]^{2}}} \\
=\frac{t_{0}}{\alpha}\left[F_{\sigma}\left(r_{\sigma}-\left(t_{0} / \alpha\right) \sigma_{0}+\left(t_{0} / \alpha\right) \delta_{0}\right)\right. \\
\left.\quad-F_{\sigma}\left(r_{\sigma}-\left(t_{0} / \alpha\right) \sigma_{0}-\left(t_{0} / \alpha\right) \delta_{0}\right)\right] \cdot(26)
\end{aligned}
$$

The equations (25) and (26) must be solved simultaneously to obtain the values for the ground-state distortions $\delta_{0}$ and $\sigma_{0}$. This is done using MathematicA. ${ }^{27}$

When we use $\alpha^{\mathrm{H}}=4.035 \mathrm{eV} / \AA$ and $V_{\sigma}(r)$ from eq. (2) with the parameters from Table II, we find that the $\sigma$ bond shrinks by $s_{0}^{\mathrm{H}}=0.128 \AA$ so that the average bond length is $r_{0}^{\mathrm{H}}=1.401 \AA$, in good agreement with experiment. However, the dimerization is $\Delta_{0}^{\mathrm{H}}=0.004 \AA$, an order of magnitude smaller than in experiment. These numbers do not change significantly when we use the linear approximation $F_{\sigma}(r) \approx-\left[K_{\sigma, 0}+K_{\sigma, 1}\left(r-r_{0}\right)\right]$ for $\left|r-r_{0}\right| \ll r_{0}$. This shows that the curvature of the force field can be ignored when we optimize the structure in the vicinity of the equilibrium distance, $r \approx r_{0}$. For given $s_{0}$ and $\Delta_{0}$, the Hückel-Peierls model requires the parameters $K_{\sigma, 0}=-5.2 \mathrm{eV} / \AA$ and $K_{\sigma, 1}=34.3 \mathrm{eV} / \AA^{2}$, corresponding to the much smaller spring constant $K_{\sigma}^{\mathrm{PA}, 1}=$ $31 \mathrm{eV} / \AA^{2}$ suggested for trans-polyacetylene. ${ }^{8}$

The comparison shows that additional terms must be included in the Hamiltonian, e.g., the Coulomb interaction between the $\pi$-electrons, that must be responsible for a large part of the bond dimerization in transpolyacetylene.

\section{Single-particle gap}

Experimentally, it is very difficult to determine the single-particle gap in trans-polyacetylene because highquality single-crystals as for PDA cannot be fabricated. Therefore, Franz-Keldysh oscillations of unbound singleparticle excitations have not been detected in electroabsorption experiments on trans-polyacetylene.

The optical absorption of polyacetylene films becomes significant above $E_{\text {onset }}=1.5 \mathrm{eV}$ and shows a peak at $E_{\text {peak }}=1.9 \mathrm{eV}$; the electro-absorption measurements display a strong signal at $\hbar \omega=1.4 \mathrm{eV} .^{28,29}$ Third-harmonic generation is large at $\hbar \omega_{\mathrm{T}}=0.6 \mathrm{eV}$ which is evidence for an excitonic state at $E_{\text {exc }}=1.8 \mathrm{eV},{ }^{30}$ as also seen in combined absorption/refraction measurements, $E_{\text {exc }}=$ 
$1.7 \mathrm{eV} .{ }^{31}$ Apparently, the linear absorption spectrum of trans-polyacetylene results from disorder-broadening of the exciton resonance. ${ }^{32}$

We assume that the exciton binding energy in transpolyacetylene is of the same order of magnitude as in other conjugated polymers such as $\mathrm{PDA}, \Delta_{s} \approx$ $0.4 \mathrm{eV} \ldots 0.5 \mathrm{eV}^{33}$ Then, the single-particle gap is estimated to be $E_{\text {gap }} \approx 2.2 \mathrm{eV} \ldots 2.3 \mathrm{eV}$. These estimates are supported by calculations of Mott-Wannier excitons for correlated electrons in one dimension. ${ }^{34}$

We compare these numbers with the predictions from the Hückel-Peierls model. The Peierls insulator has the single-particle gap

$$
\begin{aligned}
E_{\text {gap }}^{\mathrm{HP}} & =2 \Delta(\pi / 2)=4 \tilde{t}_{0} e^{\sigma_{0}} \sinh \left(\delta_{0}\right) \\
& =4 t_{0} \exp \left[-\left(r_{\sigma}-r_{0}-s_{0}\right) \alpha / t_{0}\right] \sinh \left(\delta_{0}\right) \\
& \approx 4 \alpha \Delta_{0} .
\end{aligned}
$$

The approximation holds because we have $r_{\sigma}-r_{0} \approx s_{0}$ and $\delta_{0}=\Delta_{0} \alpha / t_{0} \ll 1$. From eq. (27) we see that the combination of $\alpha^{\mathrm{H}} \approx 4 \mathrm{eV} / \AA$ with the experimental value $\Delta_{0}=0.04 \AA$ leads to a Hückel-Peierls gap of $E_{\text {gap }}^{\mathrm{HP}} \approx 0.64 \mathrm{eV}$. Apparently, the Peierls gap alone cannot account for the observed single-particle gap in transpolyacetylene.

\section{Hückel-Hubbard-Ohno model}

\section{Hamiltonian}

The Hückel-Hubbard-Ohno Hamiltonian includes the Coulomb interaction of the $\pi$-electrons in the HubbardOhno approximation,

$$
\begin{aligned}
\hat{H}^{\mathrm{HHO}}= & \hat{H}^{\mathrm{H}}+U \sum_{l}\left(\hat{n}_{l, \uparrow}-1 / 2\right)\left(\hat{n}_{l, \downarrow}-1 / 2\right) \\
& +\frac{1}{2 \epsilon_{d}} \sum_{l \neq m} V_{l, m}\left(\hat{n}_{l}-1\right)\left(\hat{n}_{m}-1\right)
\end{aligned}
$$

with the Hückel part $\hat{H}^{\mathrm{H}}$ from eq. (19) and

$$
V_{l, m}=V\left(\left|\vec{r}_{l}-\vec{r}_{m}\right|\right)
$$

with $V(x)$ from eq. (9). The carbon atoms are at the positions $\vec{r}_{l}$ of a zig-zag chain in the $x$-y-plane as is shown in Fig. 3. We optimize such trans-polyacetylene geometry numerically as will be discussed briefly below.

\section{Numerical procedure}

We employ the density-matrix renormalization group (DMRG) method ${ }^{35,36}$ together with the dynamic block state selection (DBSS) approach, ${ }^{37,38}$ to calculate the ground-state energy and excited-state energies for the Hückel-Hubbard-Ohno model for up to $L_{\mathrm{C}}=66$ carbon atoms $\left(L_{C}=2 L+2\right)$ for fixed bond parameters. We

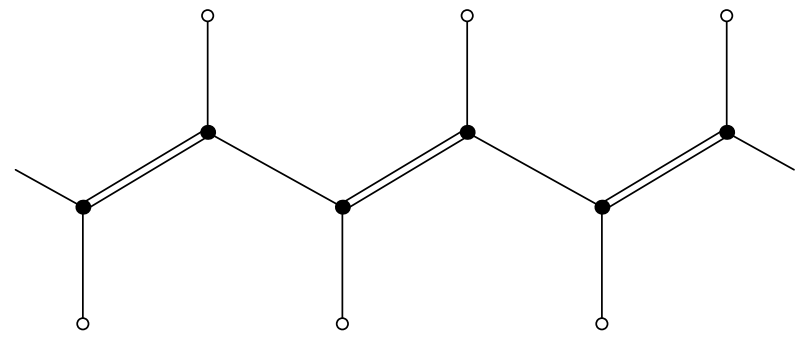

FIG. 3. Planar and unflexed trans-polyacetylene $(\mathrm{CH})_{x}$ in the ideally dimerized zig-zag Lewis structure with alternating double and single bonds between the carbon atoms (black dots). Linked to each carbon atoms are the hydrogen atoms (white circles) on alternating sides of the chain.

use a fixed-point recursion method to optimize the geometry. ${ }^{5}$ In this procedure, DMRG is called iteratively, thus playing the role of a kernel function. The electronic Hamiltonian for a fixed geometry is solved by the DMRG, and expectation values of the required operators are determined from the obtained wavefunction. These quantities are used to minimize the total energy comprising the electronic contribution and the lattice potential energy. This geometrical optimization terminates when the energy difference between two subsequent iteration steps gets below an a-priori defined threshold that we set to $10^{-4} \mathrm{eV}$. A more detailed description will be given in a subsequent paper. ${ }^{39}$

In addition, we employ the force field

$$
F_{\sigma}(r)=-\left[K_{\sigma, 0}+K_{\sigma, 1}\left(r-r_{0}\right)\right],
$$

and start our analysis with the parameter set motivated in Sect. III, $K_{\sigma, 0}=-4.7 \mathrm{eV} / \AA$ and $K_{\sigma, 1}=50 \mathrm{eV} / \AA^{2}$. Moreover, we show data for an improved parameter set, $K_{\sigma, 0}=-4.8 \mathrm{eV} / \AA$ and $K_{\sigma, 1}=42 \mathrm{eV} / \AA^{2}$. For a comparison with our earlier study on polydiacetylene, we fix $U=6 \mathrm{eV}$ and $V=3 \mathrm{eV}$. Furthermore, the dielectric screening of the surrounding medium is incorporated using the dielectric constant $\epsilon_{d}=2.3$ for the long-range part of the Coulomb interaction. Moreover, we use $t_{0}=2.5 \mathrm{eV}, \alpha=4.0 \mathrm{eV}$.

\section{Bond lengths}

The results for the average bond length $r_{0}^{\mathrm{HHO}}$ are shown in Fig. 4. As for the non-interacting case, the Hückel-Hubbard-Ohno model almost perfectly describes the bond-length reduction from $r_{\sigma}$ to $r_{0}$. We find $r_{0}^{\mathrm{HHO}, 1}=1.397 \AA$ for our first parameter set $\left(K_{\sigma, 0}=\right.$ $\left.-4.7 \mathrm{eV} / \AA, K_{\sigma, 1}=50 \mathrm{eV} / \AA^{2}\right)$ as motivated in Sect. III, and $r_{0}^{\mathrm{HHO}, 2}=1.399 \AA$ for the improved, second parameter set $\left(K_{\sigma, 0}=-4.8 \mathrm{eV} / \AA, K_{\sigma, 1}=42 \mathrm{eV} / \AA^{2}\right)$, in very good agreement with experiment, $r_{0}=1.40 \AA$. Now that $r_{0}^{\mathrm{H}, 1}=r_{0}^{\mathrm{H}, 2}=1.391 \AA$ for $\alpha=4.0 \mathrm{eV} / \AA$, we see that the electron-electron interaction and the parameter $K_{\sigma, 1}$ 


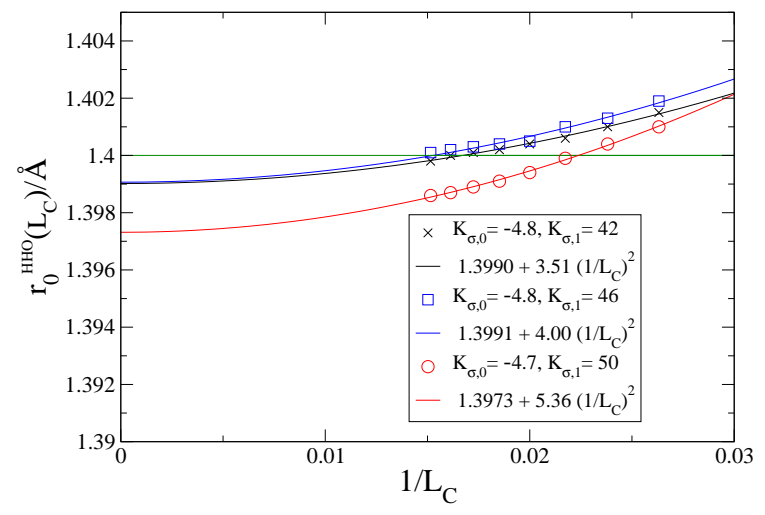

FIG. 4. (Color online) Average bond length $r_{0}^{\mathrm{HHO}}\left(L_{C}\right)$ in Angstrøm as a function of the inverse system size $1 / L_{C}$ in the Hückel-Hubbard-Ohno model with $\alpha=4.0 \mathrm{eV} / \AA$, $t_{0}=2.5 \mathrm{eV}$, and a linear $\sigma$-bond force field $F_{\sigma}(r)$ with $K_{\sigma, 0}=-4.7 \mathrm{eV} / \AA, K_{\sigma, 1}=50 \mathrm{eV} / \AA^{2}$ (red circles), $K_{\sigma, 0}=$ $-4.8 \mathrm{eV} / \AA, K_{\sigma, 1}=46 \mathrm{eV} / \AA^{2}$ (blue squares), and $K_{\sigma, 0}=$ $-4.8 \mathrm{eV} / \AA, K_{\sigma, 1}=42 \mathrm{eV} / \AA^{2}$ (black crosses). The experimental value $r_{0}=1.40 \AA$ is shown by a horizontal line, the quadratic extrapolation lines are included as a guide to the eye.

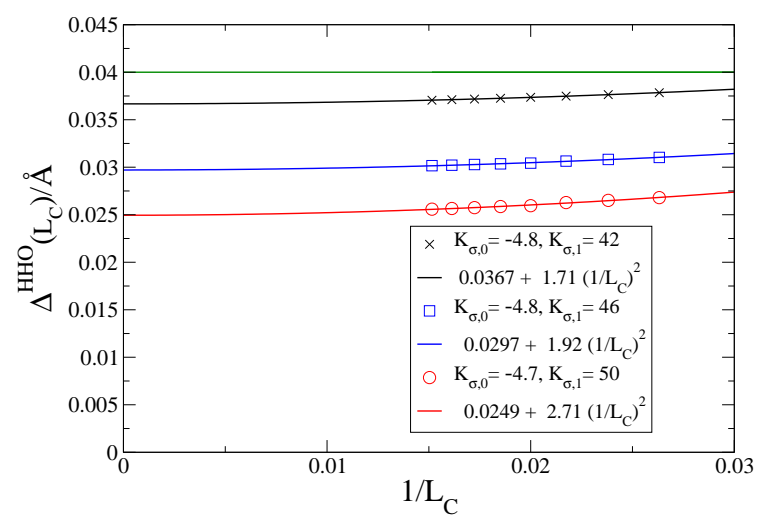

FIG. 5. (Color online) Dimerization $\Delta_{0}^{\mathrm{HHO}}\left(L_{C}\right)$ in Angstrøn as a function of the inverse system size $1 / L_{C}$ in the Hückel Hubbard-Ohno model with $\alpha=4.0 \mathrm{eV} / \AA$, $t_{0}=2.5 \mathrm{eV}$, an a linear $\sigma$-bond force field $F_{\sigma}(r)$ with $K_{\sigma, 0}=-4.7 \mathrm{eV} / \AA$ $K_{\sigma, 1}=50 \mathrm{eV} / \AA^{2}$ (red circles), $K_{\sigma, 0}=-4.8 \mathrm{eV} / \AA, K_{\sigma, 1}=$ $46 \mathrm{eV} / \AA^{2}$ (blue squares), and $K_{\sigma, 0}=-4.8 \mathrm{eV} / \AA, K_{\sigma, 1}=$ $42 \mathrm{eV} / \AA^{2}$ (black crosses). The experimental value $\Delta_{0}=$ $0.04 \AA$ is shown as a horizontal line, the quadratic extrap olation lines are included as a guide to the eye.

do not significantly influence the average bond length. Moreover, the analysis of the bond potential $V_{\sigma}(r)$ provides a good estimate of $K_{\sigma, 0}$.

In contrast, the dimerization sensitively depends on the parameter for the $\sigma$-bond spring constant $K_{\sigma, 1}$, see Fig. 5. Moreover, the estimate for $K_{\sigma, 1}=50 \mathrm{eV} / \AA^{2}$ as motivated in Sect. III leads to a too small dimerization even in the presence of the electron-electron in- teraction. Indeed, we find $\Delta_{0}^{\mathrm{HHO}, 1}=0.025 \AA$ for the parameter set $\left(K_{\sigma, 0}=-4.7 \mathrm{eV} / \AA, K_{\sigma, 1}=50 \mathrm{eV} / \AA^{2}\right)$, as compared to $\Delta_{0}^{\mathrm{H}, 1}=0.006 \AA$ from the bare Hückel model and $\Delta_{0}=0.04 \AA$ from experiment. Apparently, the electron-electron interaction substantially increases the dimerization. For the first parameter set, the dimerization in presence of the Hubbard-Ohno interaction is by a factor five larger than the Peierls contribution alone.

To obtain a better agreement with the experimentally observed dimerization, we address the improved parameter set $\left(K_{\sigma, 0}=-4.8 \mathrm{eV} / \AA, K_{\sigma, 1}=42 \mathrm{eV} / \AA^{2}\right)$. We find $\Delta_{0}^{\mathrm{HHO}, 2}=0.037 \AA$, as compared to $\Delta_{0}^{\mathrm{H}, 2}=0.016 \AA$ and $\Delta_{0}=0.04 \AA$. The value for the dimerization now agrees with the experimental value within experimental error bars. The electron-electron interaction still is decisive for the dimerization in trans-polyacetylene because the electronic contribution to the dimerization is a factor 1.4 larger than the Peierls contribution.

\section{Band gaps}

The Hückel-Hubbard-Ohno results for the exciton energy and the single-particle gap are shown in Figs. 6 and 7 , respectively.

The exciton energies extrapolate to $E_{\mathrm{exc}}^{\mathrm{HHO}, 1}=1.64 \mathrm{eV}$ and $E_{\mathrm{exc}}^{\mathrm{HHO}, 2}=1.82 \mathrm{eV}$, within the experimental bounds $1.5 \mathrm{eV}<E_{\text {exc }}<1.9 \mathrm{eV}$. The value from the improved parameter set is in good agreement with the prediction from third-harmonic generation. ${ }^{30}$ The position of the exciton energy sensitively depends on the force-field parame-

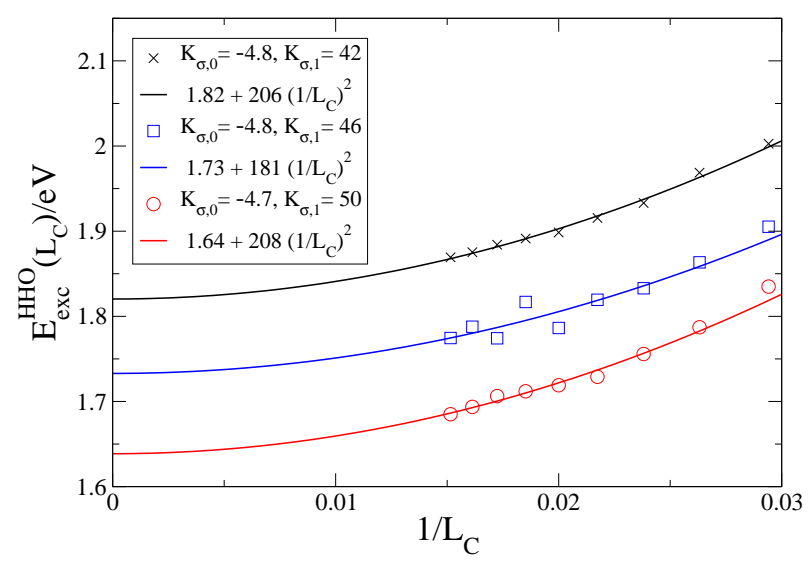

FIG. 6. (Color online) Exciton energy $E_{\text {exc }}\left(L_{C}\right)$ in eV as a function of the inverse system size $1 / L_{C}$ in the HückelHubbard-Ohno model with $\alpha=4.0 \mathrm{eV} / \AA, t_{0}=2.5 \mathrm{eV}$, and a linear $\sigma$-bond force field $F_{\sigma}(r)$ with $K_{\sigma, 0}=-4.7 \mathrm{eV} / \AA$, $K_{\sigma, 1}=50 \mathrm{eV} / \AA^{2}$ (red circles), $K_{\sigma, 0}=-4.8 \mathrm{eV} / \AA, K_{\sigma, 1}=$ $46 \mathrm{eV} / \AA^{2}$ (blue squares), and $K_{\sigma, 0}=-4.8 \mathrm{eV} / \AA, K_{\sigma, 1}=$ $42 \mathrm{eV} / \AA^{2}$ (black crosses). The quadratic extrapolation lines are included as a guide to the eye. 


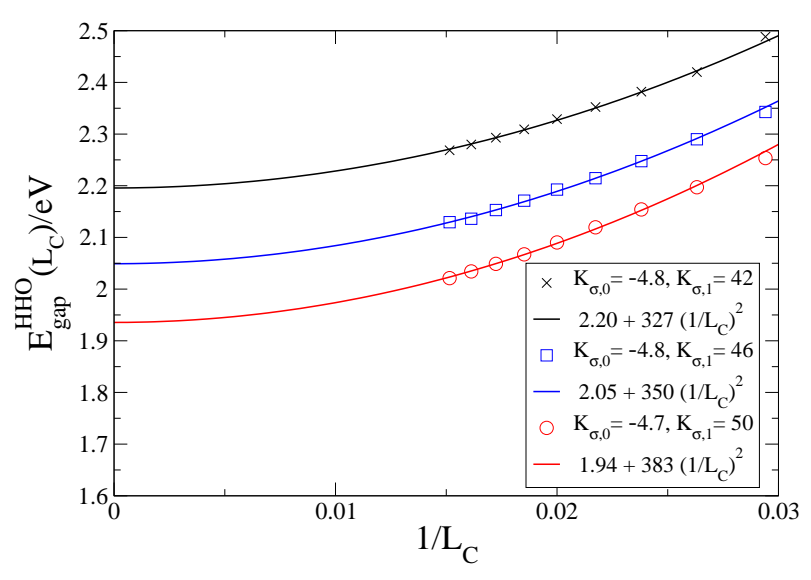

FIG. 7. (Color online) Single-particle gap $E_{\text {gap }}\left(L_{C}\right)$ in eV as a function of the inverse system size $1 / L_{C}$ in the HückelHubbard-Ohno model with $\alpha=4.0 \mathrm{eV} / \AA$, $t_{0}=2.5 \mathrm{eV}$, and a linear $\sigma$-bond force field $F_{\sigma}(r)$ with $K_{\sigma, 0}=-4.7 \mathrm{eV} / \AA$, $K_{\sigma, 1}=50 \mathrm{eV} / \AA^{2}$ (red circles), $K_{\sigma, 0}=-4.8 \mathrm{eV} / \AA, K_{\sigma, 1}=$ $46 \mathrm{eV} / \AA^{2}$ (blue squares), and $K_{\sigma, 0}=-4.8 \mathrm{eV} / \AA, K_{\sigma, 1}=$ $42 \mathrm{eV} / \AA^{2}$ (black crosses). The quadratic extrapolation lines are included as a guide to the eye.

ter $K_{\sigma, 1}$. The corresponding results for the single-particle gap are $E_{\text {gap }}^{\mathrm{HHO}, 1}=1.94 \mathrm{eV}$ and $E_{\text {gap }}^{\mathrm{HHO}, 2}=2.20 \mathrm{eV}$. The binding energy of the exciton $\Delta_{s}=E_{\text {gap }}-E_{\text {exc }}$ is predicted to be $\Delta_{s}^{\mathrm{HHO}, 1}=0.30 \mathrm{eV}$, and $\Delta_{s}^{\mathrm{HHO}, 2}=0.38 \mathrm{eV}$, in agreement with experiment. ${ }^{33}$ Apparently, the binding energy $\Delta_{s}$ increases slowly with decreasing force-field parameter $K_{\sigma, 1}$.

\section{CONCLUSIONS}

In this work we investigated the $\sigma$-bonds and $\pi$ bonds in ethane $\left(\mathrm{H}_{3} \mathrm{C}-\mathrm{CH}_{3}\right)$, ethene (a.k.a. ethylene, $\mathrm{H}_{2} \mathrm{C}=\mathrm{CH}_{2}$ ), and ethyne (a.k.a. acetylene, $\mathrm{HC} \equiv \mathrm{CH}$ ) as a function of the carbon-carbon distance $r$. We demonstrated that the $\pi$-bonds in ethene and ethyne can be described using the Hückel model with the potential $V_{\sigma}(r)$ from the $\sigma$-bond.

The bond lengths and spring constants in equilibrium agree with the data from quantum chemistry within a margin of a few percent. The comparison provides a robust estimate for the value of the Peierls coupling, $\alpha=4 \mathrm{eV} / \AA$, for a given electron transfer $t_{0}=2.5 \mathrm{eV}$ at carbon-carbon distance $r_{0}=1.4 \AA$. Unfortunately, the parameters of the Hubbard-Ohno interaction cannot be determined from dimers or short polyenes because the Ohno interaction is essentially constant for small distances. Therefore, we choose $U=6 \mathrm{eV}$ and $V=3 \mathrm{eV}$ as derived from the the analysis of excited states in polydiacetylene. ${ }^{5}$

We tested the Hückel-Hubbard-Ohno model for transpolyacetylene with $V_{\sigma}(r)$ as backbone potential. Close to the average bond length $r_{0}$, we may linearize the $\sigma$ bond force $F_{\sigma}(r)=-V_{\sigma}^{\prime}(r)=-\left[K_{\sigma, 0}+K_{\sigma, 1}\left(r-r_{0}\right)\right]$ when we determine the structure. The $\sigma$-bond repulsion balances the lattice contraction induced by the itinerant $\pi$-electrons. We find that the size of the average bond contraction is mostly determined by the constant term $K_{\sigma, 0}$ and the Peierls coupling $\alpha$ whereas the Coulomb interaction and the linear term $K_{\sigma, 1}$ are fairly unimportant for the average bond length. Moreover, the optimal value for $K_{\sigma, 0}$ is very well predicted by $V_{\sigma}(r), K_{\sigma, 0}^{(1)}=-5.2 \mathrm{eV} / \AA$ while our analysis for transpolyacetylene suggests $K_{\sigma, 0}^{(2)}=-4.8 \mathrm{eV} / \AA$.

The dimerization $\Delta_{0}$ in trans-polyacetylene is triggered to a large part by the Coulomb interaction. Naturally, the dimerization strength sensitively depends on the size of the 'spring constant' $K_{\sigma, 1}$. We find that the value obtained from the analysis of the $\sigma$-bond potential, $K_{\sigma, 1}^{(1)}=54 \mathrm{eV} / \AA^{2}$, is too large. For an agreement with experimental data in trans-polyacetylene, we propose to use the smaller value $K_{\sigma, 1}^{(2)}=42 \mathrm{eV} / \AA^{2}$, in agreement with empirical values for benzene, $K_{\sigma}^{\text {ben }}=41.3 \mathrm{eV} / \AA^{2}$, that has the same average bond length. ${ }^{12}$ Our value is considerably larger than $K_{\sigma}^{\mathrm{PA}, 1}=31 \mathrm{eV} / \AA^{2}$ proposed for trans-polyacetylene in Ref. [8] and closer to the value suggested in Ref. [11], $K_{\sigma}^{\mathrm{PA}, 2}=46 \mathrm{eV} / \AA^{2}$.

Given these parameter sets, we calculated the energy for elementary excitations in trans-polyacetylene. We find an exciton with substantial binding energy, $\Delta_{s} \approx$ $0.4 \mathrm{eV}$, in agreement with experiments for $\pi$-conjugated materials. ${ }^{33}$ The parameter $K_{\sigma, 1}$ mildly affects the exciton binding energy but determines the energetic position of the exciton. For our second, optimal parameter set $\left[t_{0}=2.5 \mathrm{eV}, \alpha=4.0 \mathrm{eV} / \AA, U=6 \mathrm{eV}, V=3 \mathrm{eV}\right.$, $\left.K_{\sigma, 0}^{(2)}=-4.8 \mathrm{eV} / \AA, K_{\sigma, 1}^{(2)}=-42 \mathrm{eV} / \AA^{2}\right]$, we find a good agreement with experiment, $E_{\text {exc }}=1.8 \mathrm{eV} .{ }^{30,31}$ The single-particle gap is found at $E_{\text {gap }}=2.2 \mathrm{eV}$. Our values for $E_{\text {exc }}$ and $E_{\text {gap }}$ also agree with the predictions from recent calculations of Mott-Wannier excitons for correlated electrons in one dimension. ${ }^{34}$

In sum, the analysis of short molecules gives a reasonable first estimate for the parameters necessary for a Hückel-Hubbard-Ohno description of conjugated polymers. To reproduce the experimental ground-state conformation, some parameters, typically the 'spring constant' at the optimal average bond length, must be adjusted by some $20 \%$. After the adjustment of these parameters, the Hückel-Hubbard-Ohno model provides a fairly good description for $\pi$-electrons in transpolyacetylene, as can be seen from the good agreement of theoretical and experimental data for the exciton energy and the exciton binding energy.

The measured optical phonon spectra provide another testing case for the Hückel-Hubbard-Ohno model. Indeed, this theoretical approach reproduces the optical phonons in trans-polyacetylene with good accuracy, as we shall show in a subsequent paper. ${ }^{39}$ 


\section{ACKNOWLEDGMENTS}

We thank G. Frenking, M. Hermann, and P. Knowles for useful discussions and for providing us their quantum- chemistry data for comparison. This research was supported in part by the Hungarian Research Fund (OTKA) under Grant Nos. K 100908 and NN 110360. L.V. was supported by the Grant Agency of the Czech Republic (Grant No 15-10279Y and Grant No. 16-12052S).
* florian.gebhard@physik.uni-marburg.de

† libor.veis@jh-inst.cas.cz

¥ legeza.ors@wigner.mta.hu

1 F. Jensen, Introduction to Computational Chemistry (Wiley, Chichester, 1999).

2 The Chemical Bond, ed. by G. Frenking and S. Shaik, vol. 1 and 2 (Wiley-VCH, Weinheim, 2014).

3 D. Baeriswyl in Conjugated Conducting Polymers, ed. by H. G. Kiess (Springer Series in Solid-State Sciences 102, Springer, Berlin, Heidelberg, 1992), p. 7.

4 W. Barford, Electronic and Optical Properties of Conjugated Polymers (Clarendon Press, Oxford, 2005, 2009).

5 G. Barcza, W. Barford, F. Gebhard, and Ö. Legeza, Phys. Rev. B 87, 245116 (2013).

${ }^{6}$ S. Kivelson, W.-P. Su, J. R. Schrieffer, and A. J. Heeger, Phys. Rev. Lett. 58, 1899 (1987); D. Baeriswyl, P. Horsch, and K. Maki, Phys. Rev. Lett. 60, 70 (1987); J. T. Gammel and D. K. Campbell, Phys. Rev. Lett. 60, 71 (1987); S. Kivelson, W.-P. Su, J. R. Schrieffer, and A. J. Heeger, Phys. Rev. Lett. 60, 72 (1987).

7 D. K. Campbell, J. T. Gammel, and E. Y. Loh, Phys. Rev. B 42, 475 (1990).

8 A. Girlando, A. Painelli, G. W. Hayden, and Z. G. Soos, Chem. Phys. 184, 139 (1994).

${ }^{9}$ G. Herzberg, Electronic spectra and electronic structure of polyatomic molecules (Van Nostrand, New York, 1966).

10 K. van Helvoort, W. Knippers, R. Fantoni, and S. Stolte, Chem. Phys. 111, 445 (1987).

11 E. Ehrenfreund, Z. Vardeny, O. Brafman, and B. Horovitz, Phys. Rev. B 36, 1535 (1987).

12 L. Goodman, A. C. Ozkabak, and S. N. Thakurt, J. Phys. Chem. 95, 9044 (1991); S. Rashev and D. C. Moule, J. Phys. Chem. A 108, 1259 (2004).

13 E. J. Mele, Mol. Chryst. Liq. Chryst. 77, 25 (1981).

14 C. S. Yannoni and T. C. Clarke, Phys. Rev. Lett. 51, 1191 (1983).

15 H.-J. Werner, P. J. Knowles, G, Knizia, F. R. Manby, and M. Schütz, WIREs Comput. Mol. Sci. 2, 242 (2012).

16 T. H. Dunning, Jr., J. Chem. Phys. 90, 1007 (1989).

17 J. B. Robinson and P. J. Knowles, J. Chem. Theory and
Comp. 8, 2653 (2012).

18 M. Hermann and G. Frenking, submitted to Chemistry A European Journal (2015).

19 M. Mottet, P. Tecmer, K. Boguslawski, Ö. Legeza, and M. Reiher, Phys. Chem. Chem. Phys. 16, 8872 (2014).

20 J. Lièvin, J. Demaison, M. Herman, A. Fayt, and C. Puzzarini, J. Chem. Phys. 134, 064119 (2011).

21 W. Krasser, A. Fadini, E. Rozemuller, and A. J. Renouprez, J. Mol. Struc. 66, 135 (1980).

${ }^{22}$ H. Fast and H. L. Welsh, J. Mol. Spec. 41, 203 (1972).

23 M.-B. Lepetit and G. M. Pastor, Phys. Rev. B 56, 4447 (1997).

24 D. Zhang, Q. Qu, C. Liu, and Y. Jiang, J. Chem. Phys. 134, 024114 (2011).

25 A. J. Heeger, S. Kivelson, J. R. Schrieffer, and W.-P. Su, Rev. Mod. Phys. 60, 781 (1988).

${ }^{26}$ F. Gebhard, K. Bott, M. Scheidler, P. Thomas, and S. W. Koch, Phil. Mag. B 75, 1 (1997).

27 Mathematica, Version 9.0 (Wolfram Research, Inc., Champaign, 2012).

28 J. Orenstein, G. L. Baker, and Z. Vardeny, J. de Phys. Colloques 44, C3-407 (1983).

29 S. D. Phillips, R. Worland, H. Yu, T. Hagler, R. Freedman, Y. Cao, V. Yoon, J. Chiang, W. C. Walker, and A. J. Heeger, Phys. Rev. B 40, 9751 (1989).

30 W.-S. Fann, S. Benson, J. M. J. Madey, S. Etemad, G. L. Baker, and F. Kajzar, Phys. Rev. Lett. 62, 1492 (1989).

31 G. Leising, Phys. Rev. B 38, 10313 (1988).

32 T. Kubo, T. Watanabe, T. Nishioka, H. Takezoe, and A. Fukuda, Jpn. J. Appl. Phys. 31, 3372 (1992).

33 M. Liess, S. Jeglinski, Z. V. Vardeny, M. Ozaki, K. Yoshino, Y. Ding and T. Barton, Phys. Rev. B 56, 15712 (1997).

34 M. Rohlfing and S. G. Louie, Phys. Rev. Lett. 82, 1959 (1999).

35 S. R. White, Phys. Rev. Lett. 69, 2863 (1992).

36 S. R. White, Phys. Rev. B 48, 10345 (1993).

37 Ö. Legeza, J. Röder, and B. A. Hess, Phys. Rev. B 67, 125114 (2003).

38 Ö. Legeza and J. Sólyom, Phys. Rev. B 70, 205118 (2004).

39 M. Timár, G. Barcza, Ö. Legeza, and F. Gebhard, in preparation. 